ORIGINAL ARTICLE

\title{
Randomised controlled trial of patient controlled analgesia compared with nurse delivered analgesia in an emergency department
}

\author{
E Evans, N Turley, N Robinson, M Clancy
}

Emerg Med J 2005;22:25-29. doi: 10.1136.emj.2002.004614

See end of article for authors' affiliations

.....................

Correspondence to:

Mr M Clancy, Emergency

Department, Southampton

General Hospital, Tremona

Road, Southampton SO16

6YD, UK; mikeclancy@

suht.nhs.uk

Accepted for publication

12 August 2003 and patient satisfaction of patient controlled analgesia (PCA)

Objective: To compare effectiveness, safety, and patient satisfaction of patient controlled analgesia (PCA)
with titrated, intravenous opioid injections for the management of acute traumatic pain in the emergency department (ED).

Methods: The study took place in the ED of a teaching hospital. Patients suffering traumatic injury requiring opioid analgesia, and meeting other inclusion criteria, were consented and randomised to either the study group or control group. The study group were given morphine through the PCA system, whereas the control group were given morphine via the conventional route of nurse titration. Pain levels were measured using a visual analogue scale. Both groups had their vital signs (blood pressure, pulse, oxygen saturations, Glasgow coma score, respiratory rate) and pain scores monitored at $0,15,30,45,60,90$, and 120 minutes, and any adverse events were noted. Patients were followed up with a questionnaire asking about their experience of pain relief in the department.

Results: 86 patients were recruited to the study, 43 in each group. There was no significant difference between the groups in terms of pain relief $(p=0.578)$ and patient satisfaction $(p=0.263)$. No severe adverse events were observed, although $20.7 \%(n=9)$ of the PCA group experienced mild sedation compared with $7 \%(n=3)$ of the control group.

Conclusions: PCA is at least as effective as titrated intravenous injections for relief of traumatic pain. It has considerable potential for use in the ED.
$P$ ain is the commonest symptom experienced by patients in the emergency department (ED), yet pain control is often sub-optimal ${ }^{1-5}$ and was confirmed by our own departmental audit.

Patient controlled analgesia (PCA) has been consistently reported to provide effective pain relief, and result in less opioid consumption and greater patient satisfaction when compared with conventional methods of analgesic delivery. ${ }^{6-11}$ PCA allows the patient to independently titrate their analgesic administration (within limits), giving the patient more control over their treatment. While PCA has been successfully used in a number of settings (postoperatively, labour suite, after burns, terminal care), and its use advocated in management of acute pain and trauma, ${ }^{12} 13$ there is little published material regarding its use in the ED. ${ }^{6} 1415$

In this setting there are a number of important differences in the patient population when comparing emergency and elective use of PCA. Patients are more likely to be fearful and apprehensive, there may be diagnostic uncertainty with often limited medical information, and little or no opportunity to observe the patient before opioid administration.

We wished to compare the effectiveness of patient controlled analgesia with our conventional means of analgesic delivery, which entails nurses giving repeated boluses of opioid on patient request in the setting of a typical ED. We intended that this was a pragmatic study.

\section{METHOD}

\section{Setting}

The emergency department, Southampton General Hospital.

This department sees 68000 new patients per annum ( 15000 under 15 years of age) serving a mixed urban and rural population of 0.5 million.

\section{Study design}

A randomised controlled trial.

Randomisation was to be undertaken independently of the researchers by a random number generator. Allocation was concealed in consecutively numbered sealed opaque envelopes.

\section{Study population}

Patients presenting to the Southampton ED aged greater than 16 years suffering from pain of traumatic origin requiring opioid analgesia based on a pain score of 7 or more were eligible and approached for consent. Patients were excluded if they were or had any of the following: age under 16; Glasgow coma score (GCS) less than 15 from any cause; moderate or severe head injury; coexistence of dementia or acute confusional state; patients with learning difficulties; patients whose first language is not English; the visually impaired or registered blind (impossible for them to use a visual analogue scale); decreased hand dexterity (may make use of PCA difficult); history of an allergy to morphine; inability to gain intravenous (IV) access; and if neither researcher was available in the department at the time of the decision to administer opioids to the patient.

\section{Outcome measures}

Primary and secondary outcome measures were studied.

\section{Primary outcome}

(1) The visual analogue scale (VAS) pain score. This is the most commonly used tool to assess pain and is sensitive

Abbreviations: ED, emergency department; PCA, patient controlled analgesisa; VAS, visual analogue scale; GCS, Glasgow coma score 
to small changes and provides a continuous variable suitable for statistical analysis.

\section{Secondary outcomes}

(1) The total amount of morphine use in both groups.

(2) Patient satisfaction to a number of questions will be assessed using a 1-5 Likert scale that has been previously used and validated in other studies. ${ }^{16}$

(3) Adverse events will be recorded by repeated measurements of the patient by direct observation by researchers.

\section{Sample size}

It has been previously reported that a $13-15 \mathrm{~mm}$ decline in VAS score was clinically significant in detecting pain relief. ${ }^{16}$ It was shown in our department (based on the evidence of three cycles of pain auditing in the Southampton ED over three years involving 750 patients) that patients reported on average a $30-40 \mathrm{~mm}$ decline in VAS score. Therefore for the purposes of our study it was decided that a difference of $20 \mathrm{~mm}$ of the mean VAS between the two patient groups would be clinically important. A sample size of 86 patients would give a power of $99 \%$ to detect a significant difference between the VAS scores of the study group and the control group.

This sample size would also give an $80 \%$ power to detect a one to two point difference in questionnaire responses. Given the rarity of adverse events, this study would be too small to make an accurate comparative analysis of adverse events in the two groups. However, an observational record of adverse events was made.

Ethical approval was sought and obtained from the local ethics committee of the Southampton University Hospitals Trust.

\section{Protocol}

After consent and randomisation, a baseline set of physiological measures (VAS, blood pressure, pulse, GCS, oxygen saturation $\left(\mathrm{SaO}_{2}\right)$, respiratory rate) was recorded for each patient, and an IV cannula inserted. The control group were given between $0-10 \mathrm{mg}$ morphine by the IV route, titrated by a nurse who had undergone the appropriate training.

\begin{tabular}{|lll|}
\hline Table 1 & Group comparability data & \\
\hline & PCA & Control \\
\cline { 2 - 3 } Number & 43 & 43 \\
\hline Mean age (y) & 60.36 & 53 \\
Sex & $18(41.9 \%)$ & $18(41.9 \%)$ \\
female & 25 total $=43$ & 25 total $=43$ \\
male & 32 & 23 \\
Type of injury & 4 & 2 \\
fracture & 2 & 1 \\
multiple injuries & 1 & 2 \\
compound fracture & 1 & 8 \\
chest injury & 1 & 1 \\
dislocation & 2 & 2 \\
blunt trauma & 0 & \\
rib and shoulder injury & 2 & \\
vertebrae/spinal injury & 0 & \\
\hline & & \\
\hline
\end{tabular}

Morphine was given at a rate of $1-2 \mathrm{mg} / \mathrm{min}$ until the nurse judged the patient to be comfortable based on the patient's responses. Patients were then asked to call for further analgesia if they needed it, and the nurse was expected to check the patient periodically, following pre-existing guidelines for the management of pain in the ED. For the PCA group, an ALARIS IVAC PCAM 5000 was set up with a $50 \mathrm{ml}$ syringe containing $50 \mathrm{mg}$ morphine sulphate in $50 \mathrm{ml}$ of normal saline. Each patient was instructed in its use and a pre-programmed protocol was started, administering a $5 \mathrm{mg}$ loading dose with a subsequent bolus dose of $1 \mathrm{mg}$ and a lock out interval of five minutes. A fixed protocol was chosen because it was felt impractical to obtain patients' weight, calculate the loading dose, and programme the PCA machine for each patient. It was felt that this may result in reduced compliance among nursing staff. A standard dose would therefore ease demands on the nursing staff, and also lower the risk of a possible administration error in the PCA group. Further sets of physiological measurements were recorded at $5,15,30,45,60,90$, and 120 minutes. The number of analgesic demands and the amount of morphine given was recorded for both groups. Patients were also given $50 \mathrm{mg}$ of cyclizine, to minimise nausea and vomiting, which can be troublesome side effects of morphine therapy.

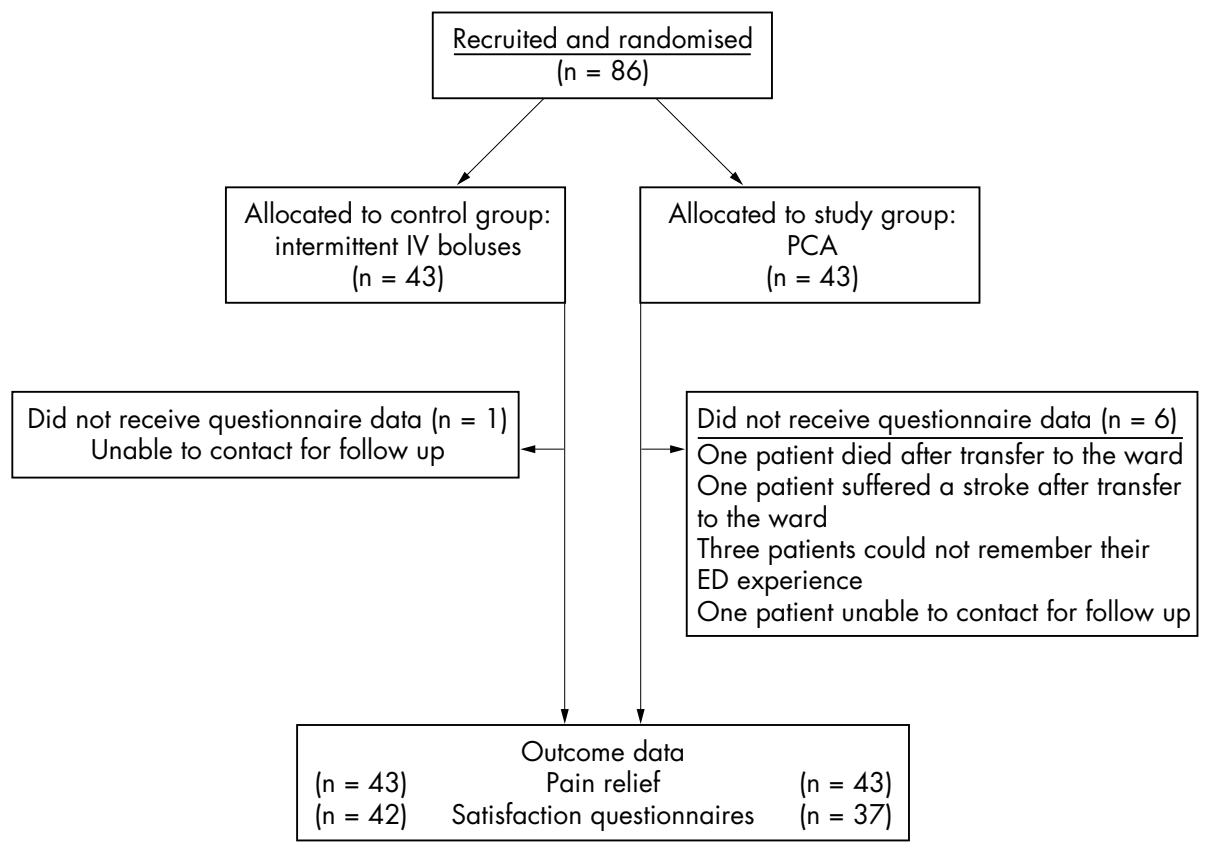

Figure 1 Flow chart. 


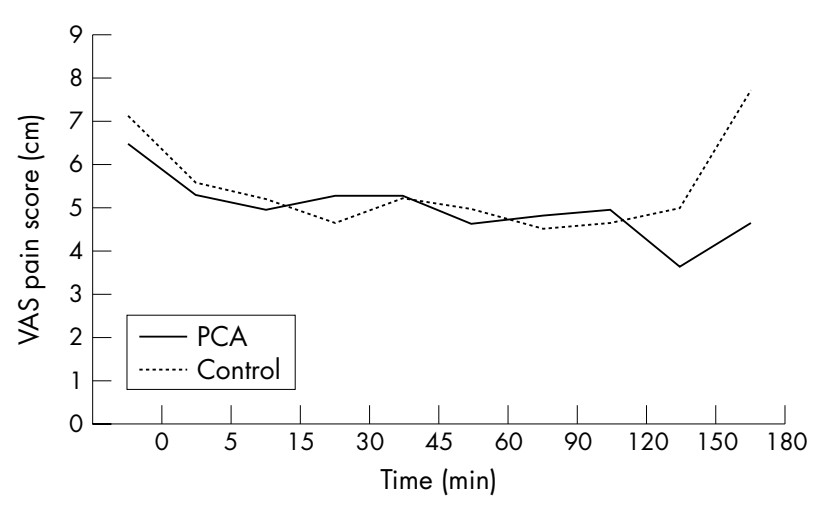

Figure 2 Line graph showing the mean VAS pain scores over time for each group.

Any significant observations or adverse events were noted, and sedation was graded as mild, moderate, or severe where appropriate. Sedation was either mild-patient is drowsy; moderate-patient is very drowsy/asleep; severe-patient is unrousable. Respiratory depression was defined as a respiratory rate less than eight breaths per minute, and hypotension as a systolic blood pressure $<100 \mathrm{~mm} \mathrm{Hg}$. Confusion was defined as GCS of 13 or 14 .

The allocated treatment was maintained until the patient was admitted to a ward or went home. Patients were free to withdraw from the study at any time. Both medical and nursing staff in charge of care for each patient, were permitted to request termination of the study if deemed necessary for clinical reasons, and also to give other analgesic medication (non-steroidal anti-inflammatory drugs, paracetamol, Entonox, regional nerve blocks) at any time during the study period. At least 12 hours after admission to a ward or discharge home, patients were contacted by the researcher and asked to complete a satisfaction questionnaire (available on line http://www.emjonline.com/supplemental). The questionnaire was either researcher administered or self administered, depending on the patient's preference, and the researchers were not blinded to which group the patients had been allocated. This was done in person if the patient was on a ward, or by post or telephone if the patient had been discharged.

\section{Statistical analysis}

Analysis of outcomes of both groups was carried out on an "intention to treat" basis. There are difficulties with statistical analyses of repeated measurements over time. When measuring at time intervals the difference between subjects may not be significant because the sample size is small, but that does not mean that there is no significant difference within the population. By testing the data at multiple time intervals, we are carrying out multiple significance tests using only a small part of the data, which means that we are losing power. The following represent possible solutions ${ }^{17}{ }^{18}$;

Table 2 Table of results obtained after area under the curve analysis of VAS pain scores

\begin{tabular}{llll}
\hline Patient & Mean & Number & SD \\
\hline PCA & -207.09 & 43 & 295.494823 \\
Control & -200.08 & 43 & 172.818020 \\
Total & -203.59 & 86 & 240.654839 \\
\hline
\end{tabular}

Table 3 Total morphine used in PCA compared with control groups

\begin{tabular}{lll}
\hline & PCA & Control \\
\hline $\begin{array}{l}\text { Mean total amount } \\
\text { of morphine used per } \\
\text { patient (mg) }\end{array}$ & $18.83 \mathrm{mg}$ & $7.65 \mathrm{mg}$ \\
$\begin{array}{l}\text { Mean time period of } \\
\text { administration }\end{array}$ & $155.5 \mathrm{~min}$ & $113.7 \mathrm{~min}$ \\
$\begin{array}{l}\text { Mean amount of } \\
\text { morphine delivered } \\
\text { over mean time }\end{array}$ & $7.26 \mathrm{mg} / \mathrm{h}$ & $4.03 \mathrm{mg} / \mathrm{h}$ \\
\hline
\end{tabular}

(1) Area under the curve

(2) Comparison of the mean VAS for the total period of observation for each patient in each group.

Therefore, VAS pain scores were analysed using these methods. The values obtained for each group were compared using an independent samples $t$ test.

Patient satisfaction questionnaire data were analysed using the Mann-Whitney U test.

A p value of 0.05 was considered significant. Results are presented with $95 \%$ confidence intervals.

\section{RESULTS}

Patients were recruited from October 2001 to May 2002. The researchers were present over that time period weekdays and weekends and on call at nights. Table 1 and figure 1 show the demographic data from both groups.

\section{PRIMARY OUTCOME}

\section{Pain relief}

VAS pain scores

Figure 2 shows the changes in the mean VAS over time. The duration of observation was anywhere up to 240 minutes and was dependent on their length of their stay.

The overall mean pain score for PCA was 4.8 (2.2 SD), and for the control group 4.8 (2.0 SD). There was no significant difference between the two groups $(p=0.578)$.

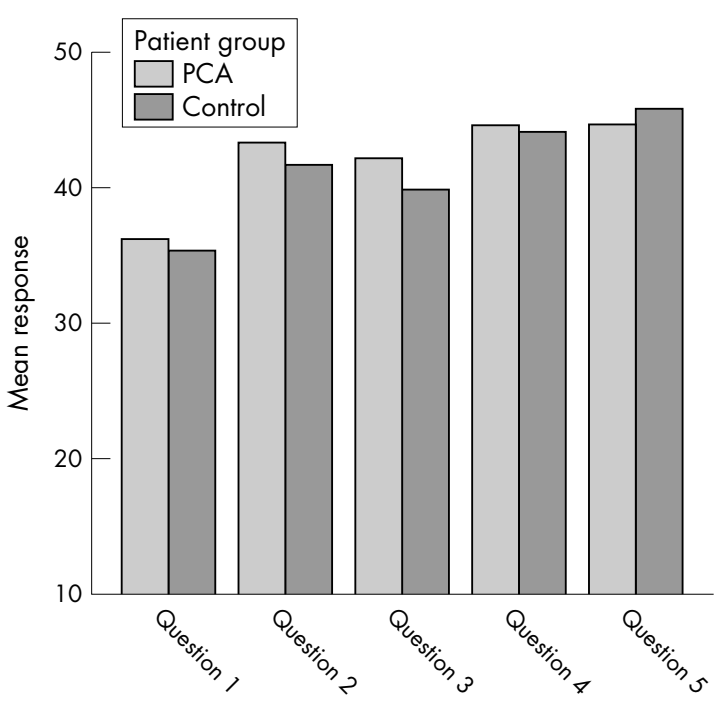

Figure 3 Bar chart illustrating the mean responses indicated by each group for each question of the satisfaction questionnaire. 


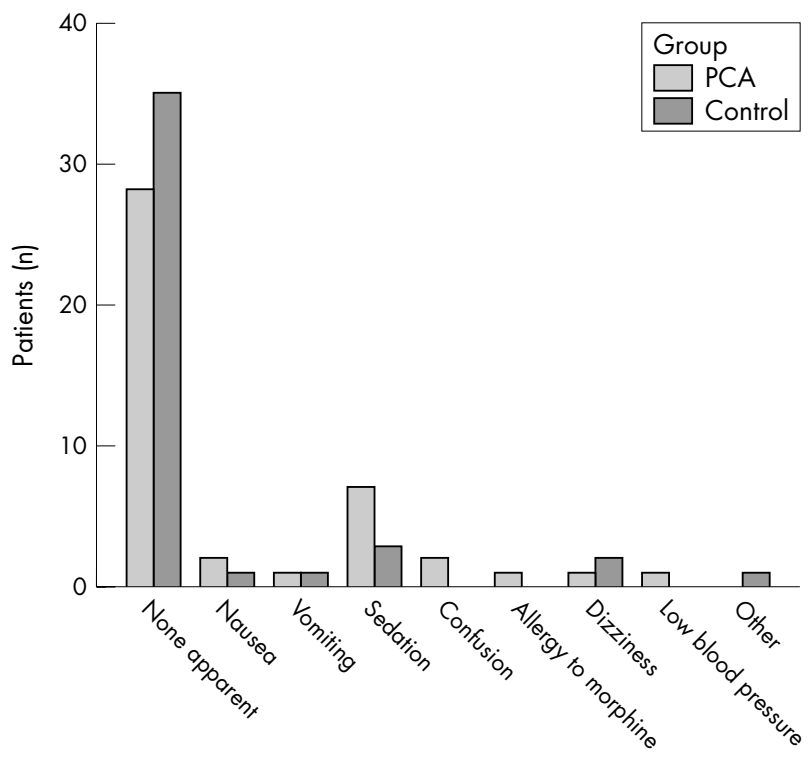

Figure 4 Bar chart showing the frequencies of the adverse events witnessed in each group.

Area under the curve

Area under the curve analysis confirmed that there was no significant difference between the two groups $(p=0.784)$ (table 2).

\section{SECONDARY OUTCOMES}

\section{Total amount of morphine used}

Table 3 gives the total amounts of morphine used in the two groups.

\section{Patient satisfaction}

There were no significant differences in the five questions asked about pain relief, the amount of pain relief, the nurse administering pain relief, and the doctor concerned about pain relief (see fig 3).

\section{Adverse events}

Figure 4 shows the frequency of adverse events. All adverse events were of mild severity, and there were no cases of respiratory depression. Overall, patients using PCA experienced more adverse events, the most common of which (in both groups) being mild sedation. There is little difference between groups for all other categories. One patient from the PCA group was described as having an allergy to morphine after developing mild thrombophlebitis around the IV cannula site after startng the PCA, although this was not verified as a true allergy.

\section{DISCUSSION}

No difference in the mean VAS pain score, or area under the curve between the two groups was identified. This was surprising given the favourable reports for PCA compared with conventional treatment at other settings. However, this trial did compare IV boluses whereas many other trials have intramuscular protocols. ${ }^{7}{ }^{10}{ }^{19}$ Our results may actually reflect that there is little difference between nurse titration of IV boluses and PCA with an accurate standard dose. The other surprising feature is the large amount of morphine given in the PCA group despite there being no difference in VAS.

Possible explanations for these findings include:

(1) It would be unreasonable to expect a standard dose of morphine to relieve the pain of a whole population. The standard administration of $5 \mathrm{mg}$ therefore is likely to fail in a proportion of patients. This would tend to reduce the PCA effect.

(2) The effect of previous audits and the presence of researchers may have influenced nursing behaviour such that they optimised their delivery of pain relief.

Possible explanations as to why twice as much morphine was given to the PCA group may include:

(1) The patients are reluctant to request analgesia from nurses, or that nurses failed to give sufficient analgesia. However, this is not shown by differences in VAS.

(2) Patients may self administer opioids for reasons other than analgesia.

(3) Other factors may be more important in the patients judgement about analgesia. The reasons for a patient to self administer are not reflected by a VAS scale.

There were no significant differences in the satisfaction questionnaires between the two groups. These results are not surprising as we would expect them to reflect the VAS results in both groups. This differs from previous studies and may reflect the greater nursing ratios in the ED setting compared with other study settings, or the reluctance of patients to criticise their treatment.

The side effect profile suggests higher sedation side effects for PCA. This may reflect the larger morphine intake in the PCA group.

It would be possible to improve the performance of PCA by using a nurse to titrate the first dose (as for the controlled group) and use PCA for subsequent maintenance with a standard bolus dose. It is conceivable that this would reduce the adverse event profile and possibly decrease the initial VAS scores in the PCA group (however this is not supported by the results of the controlled group's initial VAS scores).

If PCA were to prove as effective as standard care with a similar adverse event profile, then the decision to use PCA would be an economic one. Although economic analysis was not a formal part of this study, the fundamental difference between the two groups is the avoidance of the nurse having to repeatedly attend the patient to assess pain relief and administer morphine. Against this is the time taken to set up the PCA. It is probable that there would be very little difference in terms of cost between the two approaches. At the moment however, patients are reliant on another person-nurse or doctor-to assess and administer their pain treatment, and receiving further boluses of analgesia may be related to the availability of those people in a busy and pressured department. PCA used to maintain analgesia would be ideal as it requires much less supervision nursing/ from medical personnel. Throughout the study nursing staff were very receptive to PCA mainly for this reason.

\section{CONCLUSION}

Efforts must continue to find the most effective and efficient method of delivering available analgesia, to optimise care of patients coming to the ED in pain. Both titrated IV boluses and PCA seem to be effective and provide good patient satisfaction, probably because they can be easily changed to treat the patient's unique experience of pain. However, with increasing demands on the ED and its staff, providing this individualised analgesic control may be better maintained by putting the patient in control throughout the duration of their stay in the department.

\section{ACKNOWLEDGEMENTS}

Alaris, for the provision of equipment. Alaris had no part in the design, execution, analysis, or write up of this study. Jonathan Goddard, Department of Medical Statistics, Southampton General 
Hospital, for statistical help and advice. The staff of the Emergency Department, Southampton General Hospital: for their continued support and enthusiasm.

\section{CONTRIBUTORS}

Emma Evans, literature review, data collection and write up. Nick Turley, literature review, data collection, and write up. Neil Robinson, core ideas and study supervision. Mike Clancy, help and advice with regards to study design and write up.

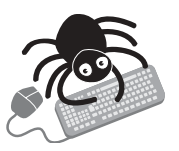

The follow up questionnaire assessing patient satisfaction with pain care received is available on line (http://www.emjonline.com/supplemental).

\section{Authors' affiliations}

E Evans, N Turley, N Robinson, M Clancy, Emergency Department, Southampton General Hospital, Southampton, UK

Funding: none.

Conflicts of interest: none declared.

\section{REFERENCES}

1 Southampton General Hospital. An audit of pain in Southampton General accident and emergency department. Southampton: Southampton General Hospital, 2000.

2 Reich M, Bodiwala GG. The use of analgesia in severe pain in the accident and emergency department. Archives of Emergency Medicine 1987;4:25-31.

3 Jantos TJ, Paris PM, Menegazzi JJ, et al. Analgesic practice for acute rthopaedic trauma pain in Costa Rican emergency department. Ann Emerg Med 1996;28:145-50.
4 Wilson JE, Pendleton J. Oligoanalgesia in the emergency department. Am J Emerg Med 1989;7:620-3.

5 Kelly AM. A process approach to improving pain management in the emergency department: development and evaluation. J Accid Emerg Med 2000;17:185-7.

6 Choiniere M, Grenier R, Paquette C. PCA-a double blind study in burns patients. Anaesthesia 1992;47:467-72.

7 White PF. Use of patient controlled analgesia for management of acute pain. JAMA 1988;256:243-7.

8 Lindley C. Overview of current development in PCA. Support Cancer Care 1994;2:319-26.

9 Chumbley GM, Hall GM, Salmon P. Why do patients feel positive about PCA? Anaesthesia 1999;54:372-92.

10 Chumbley GM, Hall GM, Salmon P. PCA-an assessment by 200 patients. Anaesthesia 1998;53:216-21.

11 Taylor NM, Hall GM, Salmon P. Patient's experiences of PCA. Anaesthesia 1996;51:525-8.

12 Rosenberg AD, Grande CM, Bernstein. Pain management and regional anaesthesia in trauma. Chicago: WB Saunders, 2000.

13 Acute Pain Management Guideline Panel. Acute pain management: operative or medical procedures and trauma. Washington: US Department of Health and Human Services, 1992.

14 Owen H, Mather LE, Rowley K. The development and clinical use of PCA Anaesthesia and Intensive Care 1988;16:437-47.

15 Duchenne J. Emergency pain management: a Canadian Association of Emergency Physicians (CAEP) consensus document. J Emerg Med 1994; 12:855-66.

16 Stahmer S, Schofer F, Marino A, et al. Do quantative changes in pain intensity correlate with pain relief and satisfaction? Acad Emerg Med 1998:5:851-7.

17 Matthews JN, Altman DG, Campbell MJ, et al. Analysis of serial measurements in medical research. BMJ 1990;300:230-5.

18 Bryant TN. The presentation of statistics. Pediatr Allergy Immunol 1998:9:108-15.

19 Shipton E, Beeton AG, Minkowitz HS. Introducing a patient controlled analgesia based acute pain relief service into Southern Africa-the first 10 months. S Afr Med J 1993;83:501-5.

20 Ballantyne JC, Carr DB, Chalmers TC, et al. Postoperative patient controlled analgesia: meta-analyses of initial randomised controlled trials. J Clin Anaesth 1993;5:182-93. 